Supersymmetry

and

Supergravity 


\section{Princeton Series in Physics}

Edited by Sam B. Treiman (Published since 1976)

Quantum Mechanics for Hamiltonians Defined as Quadratic Forms by Barry Simon

Lectures on Current Algebra and Its Applications by Sam B. Treiman, Roman Jackiw; and David J. Gross

The Many-Worlds Interpretation of Quantum Mechanics edited by B. S. Dewitt and N. Graham

Homogeneous Relativistic Cosmologies by Michael P. Rvan, Jr., and Lawrence C. Shepley

The $\mathrm{P}(\mathrm{f}) 2$ Euclidean (Quantum) Field Theory by Barry Simon

Studies in Mathematical Physics: Essays in Honor of Valentine Bargmann edited by Elliott H. Lieb,

B. Simon, and A. S. Wightman

Convexity in the Theory of Lattice Gasses by Robert B. Israel

Works on the Foundations of Statistical Physics by N. S. Krylov

Surprises in Theoretical Physics hy Rudolf Peierls

The Large-Scale Structure of the Universe by P.J.E. Peebles

Statistical Physics and the Atomic Theory of Matter, From Boyle and Newton to Landau and

Onsager by' Stephen G. Brush

Quantum Theory and Measurement edited by. John Archibald Wheeler and Wojciech Hubert Zurek

Current Algebra and Anomalies by Sam B. Treiman. Roman Jackiw, Bruno Zumino, and Edward

Witten

Quantum Fluctuations by E. Nelson

Spin Glasses and Other Frustrated Systems $h \cdot$. Dehashish Chow'dhury (published in cooperation with World Scientific Publishing Co. Pte. Ltd., Singapore)

Weak Interactions in Nuclei by Barry R. Holstein

Large-Scale Motions in the Universe: A Vatican Stúdy Week edited by Vera C. Rubin and George $V$. Colne. S.J.

Instabilities and Fronts in Extended Systems by Pierre Collet and Jean-Pierre Eckmann

More Surprises in Theoretical Physics by Rudolf Peierls

From Perturbative to Constructive Renormalization hy Vincent Rivasseau

Supersymmetry and Supergravity ( $2 \mathrm{~d}$ ed.) by Julius Wess and Jonathan Bagger

Maxwell's Demon: Entropy, Information, Computing edited by Harvey' S. Leff and Andrew F. Rer

Introduction to Algebraic and Constructive Quantum Field Theory by John C. Baez. Irving E. Segal, and Zhengfang Zhou

Principles of Physical Cosmology by P.J.E. Peebles

Scattering in Quantum Field Theories: The Axiomatic and Constructive Approaches by Daniel lagolnitzer

QED and the Men Who Made It: Dyson, Feynman, Schwinger, and Tomonaga by Silvan S. Schweber

The Interpretation of Quantum Mechanics by Roland Omnes

Gravitation and Inertia by Ignazio Ciufolini and John Archibald Wheeler

The Dawning of Gauge Theory hy Lochlainn O'Raifeartaigh

The Theory of Superconductivity in the High- $T_{C}$ Cuprates $h y$ P. W. Anderson

Master of Modern Physics: The Scientific Contributions of H. A. Kramers by Dirk ter Haar

Critical Problems in Physics edited hy Val L. Fitch, Daniel R. Marlow, and Margit A. E. Dementi 


\title{
Supersymmetry \\ and Supergravity
}

SECOND EDITION,

REVISED AND EXPANDED

\author{
by \\ Julius Wess \\ and \\ Jonathan Bagger
}

Princeton Series in Physics

Princeton University Press

Princeton, New Jersey 
Copyright $(\odot 1992$ by Princeton University Press

Published by Princeton University Press, 41 William Street,

Princeton, New Jersey 08540

In the United Kingdom: Princeton University Press, Chichester, West Sussex

All Rights Reserved

\section{Library of Congress Cataloging-in-Publication Data}

Wess, Julius.

Supersymmetry and supergravity / by Julius Wess and Jonathan

Bagger. - 2nd rev. and expanded ed.

p. $\quad \mathrm{cm}$.

ISBN 0-691-08556-0. - ISBN 0-691-02530-4 (pbk.)

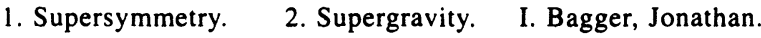

II. Title.

QC174.17.S9W47 $1991 \quad 530.1^{\prime} 43-d c 20 \quad 90-26372$

Princeton University Press books are printed on acid-free paper and meet the guidelines for permanence and durability of the Committee on Production Guidelines for Book Longevity of the

Council on Library Resources

$$
\text { http://pup.princeton.edu }
$$

Printed in the United States of America 
To Traudi 
